of controversies in an area characterized, as is much of science, by a complex mixture of status-seeking and truth-seeking on the part of individual scientists. He does not avoid making hard and sometimes harsh judgements. His purpose is to clarify the issues, but the petulance with which he insists that his own contributions have been unappreciated and misinterpreted is, sadly, likely to stimulate, rather than resolve, future controversies.

The goal of science is always to seek generalizations, but Brown clearly demonstrates that no single hypothesis is adequate to explain the evolution of helping and communal breeding in all species or even the evolution of all components of the social organization of a single species. Evolutionary pathways to sociality have been diverse. Different species have been influenced by different social and environmental factors.

Comprehensive though the book is, more attention could have been paid to species that appear to fulfil the preconditions for the evolution of helping and communal breeding but do not exhibit such behaviour. We often learn as much from an analysis of what has not happened as we do from studying what has happened. In any case, every student of evolutionary theory, sociobiology or behavioural ecology should read and study this book very carefully. It will be the standard reference on the subject for many years.

Gordon H. Orians is a Professor in the Department of Zoology, University of Washington, Seattle, Washington 98195, USA.

\section{Models and games}

\section{Kendall Preston, $\mathrm{Jr}$}

Cellular Automata Machines: A New Environment for Modeling. By Tommaso Toffoli and Norman Margolus. MIT Press: 1987. Pp.259. \$30, £26.95.

Four decades ago John von Neumann, the famous mathematician, became interested in the power of digital computation. At that time there were perhaps two fullyconfigured digital computers in the world. These were not much more than today's programmable hand calculators. Each used a single ALU (Arithmetic Logic Unit), stored a relatively small program and executed instructions slowly.

Von Neumann, however, with his extraordinary vision of the future, began to study the computational capabilities of thousands of ALUs interconnected in a massive planar array where each ALU had direct connections to its neighbours. He called such arrays 'cellular automata'. With von Neumann's premature death, and the impossibility of constructing cellular automata with the technology of the time, cellular automata studies then languished.

In the $1950 \mathrm{~s}$, however, Moore and his colleagues at the United States National Bureau of Standards, Unger at Bell Telephone Laboratories, Dinneen and Selfridge at the Massachusetts Institute of Technology (MIT), and Golay and his colleagues at Perkin-Elmer recognized that simulators of cellular automata could be built using fast digital hardware employing the transistor. This was done and, by the 1960 s, important research was being carried out with what were called 'cellular logic machines'.

At the same time, work by McCormick and Skolnick at the University of Illinois led to the first true cellular automaton Illiac IV, a small array $(8 \times 8)$ of 64 computing elements. In Britain, International Computers Ltd and University significant cellular automata, namely the $32 \times 32$ Distributed Array Processor and the $96 \times 96$ Cellular Logic Image Processor, respectively, in the late 1970 s.

In the 1980s there have been further advances, primarily in the United States. There are now the $128 \times 128$ Massively Parallel Processor of the National Aeronautical and Space Agency, the $256 \times 256$ Connection Machine of the Thinking Machines Corp. and the $216 \times 384$ Geometric Array Parallel Processor of Martin Marietta.

Unfortunately, the book under review does not concern itself with any of the above, except for brief mention of the Connection Machine's capability in Instead, the authors concentrate on the cellular automata simulator CAM-6 which they have developed at MIT. This is not to disparage the book, the contents of which are quite fascinating to the aficionado of the cellular automaton, but simply to indicate that the title is a gross misnomer. A better title for the first part of the book would be "Cellular Automata Games for CAM-6" and, for the latter part, "Modeling Physics on CAM-6".

The book has its origins in Fredkin's research at MIT and also in the cellular automata game called 'Life' devised by the mathematician John Horton Conway - both originating in the $1960 \mathrm{~s}$. These stimuli led to intensive studies of cellular automata games at MIT, not only using Conway's rule but also many other rules devised by Fredkin's colleagues and students in the Information Mechanics Group. Much of this work is brilliant and it is a pleasure to find it thoroughly documented, with detailed rules and profuse illustrations, in this book. The authors are fascinated by the patterns generated by displaying the contents of the processing elements of the cellular College London produced the first really running fluid-dynamics computations. automaton on the computer screen, both in black and white and in colour. For example, of the display generated by the rule called "TUBE-WORMS", they write: "these animals have plumes that look like delicate flowers, but at the slightest disturbance the 'flower' retracts into a tube and waits a good fraction of minute before coming out again".

Of more practical interest is the latter half of the book which deals with the use of CAM-6 in "modeling of the laws of physics". In this regard the book is not particularly analytical - there is little or no mathematical development - although the heat equation, the diffusion equation and the Navier-Stokes equation do put in an appearance. The illustrations provided do not convince me that the physical models generated by programming CAM- 6 are exact. In fact, the authors state that "the gases described by a cellular automaton rule . . . obey the Navier-Stokes equation approximately". The reader is not told how good this approximation is, although we are referred to the literature on hexagonal tessellation cellular automata and are told that exact solutions have been obtained. (CAM-6 is not hexagonal but uses the square tessellation.) This, of course, is of great significance and there is a growing interest in applying cellular automata to research on fluid dynamics. General studies of this kind, however, require three-dimensional analyses and, unfortunately, the book mentions work on three-dimensional automata (such as that being carried out at Carnegie-Mellon University) only peripherally.

The colourful pictures are attractive, but otherwise this is not a book for the casual reader. To understand the multitude of CAM- 6 rules presented, one must learn the vagaries of the programming language FORTH. An appendix is provided for this purpose, but is in some ways inadequate; the authors' use of the FORTH "case statement", first introduced on page 20 , is not properly explained until page 40 . But these are minor annoyances to the dedicated reader who is willing to read and re-read to get at the meat of this work.

The book is well referenced with both current and historical material, especially as regards recent applications to physical modelling. Although there are shortcomings, it still deserves serious attention by the rapidly growing community of computer scientists and physicists who now work with cellular automata and cellular automata simulators - not to mention the 'computer artist' who simply wants to sit enthralled watching tubeworms evolve on the computer display.

Kendall Preston, Jr is a Professor in the Department of Electrical and Computer Engineering, Carnegie-Mellon University, 5000 Forbes Avenue, Pittsburgh, Pennsylvania 15213, USA. 